\title{
Socioeconomics and Politics Affecting the Japanese Neurosurgeon
}

\author{
James I. AUSMAN ${ }^{* * *}$ and Tadashi SHIBUYA*** \\ ${ }^{*}$ Department of Neurosurgery, University of Illinois at Chicago, Chicago, Illinois, U.S.A.; **Editor, \\ Surgical Neurology; ***Department of Emergency and Critical Care Medicine, \\ Nihon University School of Medicine, Tokyo
}

Key words: socioeconomics, politics, managed care, neurosurgeons

\section{What is Happening to Neurosurgeons Everywhere}

Ask almost any neurosurgeon in the developed world what his or her main concern is. It is the effect of socioeconomics and politics on his life, not science or the latest way to treat an aneurysm or lumbar disc. Why? Because there are too many neurosurgeons competing with each other, the amount they are being paid for their work is decreasing, and their incomes are falling after years of study for a career they believed would provide economic security. Other specialties are competing for the cases that used to be the sole province of neurosurgery; malpractice threats are clouding physicians' lives. Neurosurgeons feel there is little they can do about these changes. In general, neurosurgeons are independent and individualistic, and unused to group organization. Their national societies often seem to be powerless to make any significant changes in the laws that will affect any of these outside forces. Therefore, many neurosurgeons have decided that they should live for the present and make as much money as they can, because the future may be worse. We can see the results of this kind of thinking in the tendency of many doctors, hospitals, administrators, and health care systems to practice medicine for money rather than for the good of the patient. In the developing world, the plight of neurosurgeons is not much better, although the problems they face may be different. What has happened to change the lives of neurosurgeons around the world? The world has changed, and is still changing.

\section{Major Changes in World Politics in the Last Half-Century}

World politics have changed in the last half of the 20th century, with the resolution of major wars; the end of the Cold War; the collapse of the communist system; the rise of individual freedom; and the challenge to autocracy locally, nationally, and internationally. As countries have prospered, more people want to become physicians and neurosurgeons, professions that engender greater respect and better financial reward than many others do.

\section{The Rise of a Global Economy and the Influence of Business}

As interaction between people and countries has increased, businesses have been challenged to compete internationally, producing quality goods at low cost. These challenges have forced countries to lower trade barriers. As businesses and governments struggle to compete in this global arena, health care spending, which they largely control, has become limited.

\section{The Introduction of Managed Care}

Rising health care costs are controlled by spending only predetermined, fixed amounts on health care, a policy which has given rise to capitation and managed care in some countries. Patients can no longer freely obtain the kind of care they desire; they must obtain permission from a "gatekeeper" physician, who ensures that the predetermined amount of money allowed for health care is spent appropriately. If too much is spent, the gatekeeper's salary is 
reduced because he or she is paid from what is left after the capitated money is spent on the patient's care. However, patients are becoming dissatisfied with this system, realizing that the managed care companies' first priority is making money, not caring for patients. The managed care "solution" was instituted because governments and businesses were involved in finding the solution, while physicians spent their time taking care of their patients and avoiding politics, which they don't like. What has been happening to the dreams of doctors under this system? Who is controlling those dreams?

\section{The Effects of the Asian Economic Collapse}

In 1998 , we saw more clearly than ever that changes in one part of the world can affect the rest of the world. The economies of Asia deteriorated as Thailand, Malaysia, and then Korea went bankrupt because of poor investments and industrial overcapacity. Investors in other Asian businesses withdrew their money in a panic, resulting in failing businesses, unemployment, and falling prices. In order to keep their factories producing and their workers employed, companies lowered their prices. Their lower-priced goods were imported by more economically stable countries, but businesses in these countries then sold less of their own products. Under these conditions, profits fell even in stable economies, and stock market values around the world tumbled as the prospect of profitable investments faded. Retirement funds, in which many physicians have invested their savings, lost value for a time as the stock markets declined.

\section{The Effect of the Failure of the Japanese Economy}

The failure of the Japanese economy has also affected people around the world, including neurosurgeons. Japan has the second largest economy in the world and is the major source of investment in Asian countries. Because Japanese banks had made some of the risky loans to other Asian businesses, they lost a great deal of money when the economies of these countries failed. The problem with Asian lending practices is that they are based on friendship rather than performance. Until very recently, there has been little movement in Japan to alter the rules governing banks that loan money to others. Now with huge public investments, the economic crisis in Japan seems to be reversing itself. This is another example of governments and businesses failing both in one country, Japan, and on a worldwide scale. These are the same organizations which are trying to solve the world's health care problems. The profession of medicine, and our patients, have suffered as a result.

\section{Impact of World Economic Failure on South America and Others}

Investors panicked by uncertainties in other economies began to withdraw their money from South American countries. Brazil, which has the largest economy in South America and the ninth largest in the world, devalued its currency, which was overvalued. The economic stability of Brazil tumbled. The other South American economies are threatened by Brazil's financial failure because of their interdependence on trade.

Businesses in the U.S.A., as well, began to lose money, as their sales in Asia declined and their profits fell. Poor investment strategies have forced one large investment company to fail, showing that the U.S.A. is not immune to the poor business practices that have affected others. The global economy has deteriorated in the last 6-12 months. This deterioration will directly affect the amount of money that will be spent on health care.

Can neurosurgeons expect more money to be available for health care as companies further restrict their health care expenses and unemployment increases, leaving workers without insurance? Will there be money for new equipment? What will the future of medicine and neurosurgery be in every country around the world under these circumstances? What strategies do you have for your future? Or do you believe these changes are beyond your control?

\section{The Collapse of the Russian Economy by Corrupted Capitalism}

As an example of how changes in one country can directly affect its physicians, let us look at what has happened in Russia. The Russian economy has also collapsed, because the country was unable to handle the challenge of a free-market economy that was introduced in the 1990s. For the Russian people, who are used to total dependence on the government, the burden of individual responsibility in a capitalistic economy has become too much. A few people who used the system for their own personal advantage have corrupted the new Russian capitalism. Is this failure of capitalism representative of what will happen as emerging nations, with little experience with democracy and freedom, are exposed to the free market? What has happened to neurosurgeons 
in Russia? They have become victims of the national and global changes.

\section{8: World Wide Political Changes}

In 1998, the President of the U.S.A. was investigated for alleged perjury and other crimes, and was impeached. The President of Russia lost power as the country's economy collapsed. The leader of Germany for 15 years, a social conservative, was defeated by a social liberal. France and other countries in Europe elected new governments that are more liberal in their social programs. These new governments promised fiscal responsibility with social generosity, a concept that appealed to voters unwilling to reduce their standard of living as a sacrifice to the national debt. This new socialistic philosophy has been called "the Third Way."

Most countries are in debt, because their governments have spent more money than they have collected from taxes; at the same time, however, politicians, in order to remain in office, are promising the people more but health care costs are being restricted to limit the national debts. Under these circumstances, what are medicine's prospects for obtaining a larger piece of the national budget?

\section{Conflicts World Wide}

In 1998, near-wars broke out in the Balkans. The threat of war between Serbia and Kosovo continues with the potential to spread to the surrounding countries and to Europe. Tensions have risen between Iran and Afghanistan, between Israel and its neighbors, between Turkey and Greece, between Turkey and Syria, and in Africa. Iraq has defied the demands of the world to allow inspections for hidden armaments. The U.S.A. launched missile attacks against terrorists in Sudan and Afghanistan and an air war against Iraq. All of these conflicts are occurring in a part of the world that controls one-third of the world's natural resources. How will these conflicts affect medicine? What happened to the price of oil when oil-producing nations restricted its sale to make more money? The rest of the world's economies went into recession.

In 1998, India and Pakistan exploded nuclear devices, thereby becoming members of the international nuclear club. They could no longer depend on the protection of the nuclear powers, which they perceived as becoming powerless, to protect either their own people or the world order. They saw no reason why they should not become members of the "nuclear club," a group of countries which had nuclear weapons and used them to assert their power while trying to prevent others from acquiring nuclear weapons and thereby gaining equal power. The threat of nuclear proliferation has spread to Middle Eastern countries which, to westerners, do not appear to be very responsible with these weapons. Many countries have broken embargoes and treaties to supply hostile countries with arms of all types. Politicians have ignored these violations the same politicians who are trying to solve the health care problems.

\section{China}

While all of these changes have been occurring, China has grown to be a powerful economic and political force in the world. By the end of 1998, because the world economic crisis had made it difficult for China to export its goods, its percentage of unemployed people rose to nearly $10 \%$ of the population. In their dissatisfaction this large group of unemployed people could threaten the Chinese communist government by promoting civil unrest. As a preventative response the Chinese government repressed the freedom of expression and placed more governmental controls on China's economic development. What will happen when China becomes the dominant trading nation in the world because of its large consumer population? Will it be able to dictate to the developed countries what changes they should make in their social structures in order to qualify for China's trade (as the U.S.A. has done to China)? Clearly, the world is changing for everyone - and for neurosurgeons, too!

\section{The Spread of the Influence of the West and the Fundamentalist Reaction}

The desire to make money - the new materialism has become more important than principles or idealism. The fundamentalist movements worldwide express a desire to adhere to "old ways," as a reaction to this newly found materialism. Yet, the compulsion to make money has seeped into every facet of peoples' lives. The influence of western culture, from blue jeans to movies, has spread around the world and has been adapted to different societies. However, this influence began to diminish after the end of the Cold War, which polarized the world, as these other societies declared their individuality from the western world. The western powers have been the last to realize that the other countries were only adapting the western ideas into their own cultures, not becoming captive to western ideology and practice, which is seen as 
flawed. Television brought the sins of western culture to the rest of the world, including the many societies that are less liberal and permissive and more respectful of each other.

\section{The Effects on Medicine, Physicians, and Neurosurgeons}

What we have witnessed in recent years is a world that had resolved global wars, but is now on the verge of small wars in many areas. We have seen the end of the Cold War and the collapse of a philosophic and economic system-communism. The influence of business and its ethics has affected our lives because of the rising importance of trade among countries. Because politicians do not want to raise taxes, and because businesses must make a profit, expenditures for health care have become restricted. The economies of many countries are failing because of poor management, as businesses and banks are failing because of poor investments. These are the same governments and businesses that have tried to solve health care problems on their own with little constructive input from physicians.

Physicians appear to be more interested in their individual survival than in their success as a group. Their national societies lack the leadership required to deal successfully with all these issues. For example, the membership in the American Medical Association has declined to only one-third of the physicians in the U.S.A. It has obviously lost its appeal to physicians as an organization that is successfully promoting their views. Neurosurgical societies around the world will also lose their members' support if they do not provide value. As neurosurgeons' incomes fall, more of them will begin to question whether they are getting their money's worth from their representative societies.

Many of the problems medicine and neurosurgery face are the same around the world. There are too many neurosurgeons and too many hospitals in which different levels of neurosurgical care are given. Every physician and hospital wants to have the latest equipment in order to compete with other physicians and hospitals, resulting in a needless waste of resources. Many hospitals do not have enough neurosurgical cases to train neurosurgeons. Governments will not allow neurosurgical societies to regulate the number of trainees. National societies seem to be unable to deal with these problems effectively and, because of this perceived failure to represent their members' interests, they do not have much support from their members. Neurosurgeons fear encroachment on their specialty by other physicians-orthopedists, vascular surgeons, plastic surgeons, and interventional neuroradiologists who are also looking for more patients because they are under similar economic threat. All of these changes are occurring worldwide, and there are very few groups trying to come up with constructive solutions. Most neurosurgeons have conceded defeat; they are depressingly hopeless about dealing with these "unsolvable" problems. Physicians, including neurosurgeons, have given their patient the practice of medicine - up for dead. Few are thinking about new, innovative therapies to save the patient's life, or their own.

\section{Solutions from Other Countries}

But in a few countries, such as Brazil, Denmark, and the U.S.A., neurosurgeons have developed ideas to help deal with the changing world. In Brazil, which suffers from an oversupply of neurosurgeons as well as an economic collapse, the neurosurgeons have come up with an answer that may be applicable to neurosurgery worldwide. After years of planning and meetings with government officials, the Brazilian neurosurgeons, led by Carlos Batista de Sousa, have received governmental approval for a plan that divides hospitals into three groups, depending on the facilities, equipment, and number of neurosurgeons each one has. Fully equipped hospitals with three or more neurosurgeons will be allowed to care for all types of cases. Hospitals with two neurosurgeons will be more limited in the neurosurgery which they can offer while those with only one neurosurgeon will be allowed to do only minimal neurosurgical procedures. Neurosurgeons must be certified by their national society. Because of this arrangement, which will save money and improve health care quality, the government doubled neurosurgeons' compensation. (If you would like to learn more, contact Carlos Batista de Sousa, Nelson Ferreira, Gilberto Machado de Almeida, or Paulo Mello.)

In Denmark, which also has too many neurosurgeons, some of those involved in resident training have agreed to merge their training programs, even though they are located in different cities. They have also agreed to concentrate specific types of cases at each center, allowing each to develop a large experience in a particular field of neurosurgery - for example, arteriovenous malformations or skull base surgery - while eliminating the expense of redundant equipment. Residents rotate through the programs, spending 3 months at each of the various centers learning about its particular specialty. The program includes non-academic institutions as well; neurosurgeons in the non-academic hospitals are 
made professors in one of the associated medical centers. With this program, patients can be given better care at lower cost, and resident education can be enhanced by taking advantage of the best of each participating institution. (Jens Haase can be contacted for more information about this innovative program.)

For several years, the two major national neurosurgical organizations in the U.S.A. have combined forces to offer practical courses, held both at the national meetings and throughout the year. Neurosurgeons learn spine surgery, instrumentation, vascular surgery, pain management, peripheral nerve surgery, and other specialty interests, allowing each to add new skills to his or her practice.

As neurosurgeons, what can you do in Japan to change the course of health care? You must apply the reasoning you developed in studying and practicing medicine. You must list the problems you face, consider the different possible solutions, and then select the best option.

\section{What are the Problems You Face in Japanese Neurosurgery?}

Number of Neurosurgeons: Are there too many neurosurgeons in Japan? According to Fukui ${ }^{13}$ there were 4550 neurosurgeons in 1997. By the year 2000 there will be 5000 . Based on a population of 135 million there will be one neurosurgeon for every 27,000 people. What will be the consequences for neurosurgeons serving such a small population? What will happen to the quality of care? Under these conditions should the training of more residents in neurosurgery be limited? Some state that this ratio of neurosurgeons to population is deceptively low because many neurosurgeons are not practicing neurosurgery but are in associated neurological disciplines. What then is the true ratio? Even if it is $1: 54,000$ do the same questions apply?

Training of Neurosurgeons: Are there too many hospitals in which residents receive training in Japan? There are 1070 hospitals approved by The Japan Neurosurgical Society for neurosurgical training. ${ }^{1)}$ In the U.S.A., which has twice the population, there are only 95 neurosurgical-training programs.

Is all of the neurosurgical training equal in these hospitals? Do neurosurgeons trained in A hospitals gain more experience than those in $\mathrm{C}$ hospitals? ${ }^{1)}$ Are neurosurgeons trained in different hospitals exposed to the same number of cases and thus equally experienced in neurosurgery?

Is there a formal approved residency education program for all residents? Are the residents who pass their board examinations equally competent to practice neurosurgery? Does the Neurosurgery Board have enough power to enforce its decisions in regard to residency training?

Number of Hospitals: Are there too many hospitals in Japan overall? Should some be closed? Would this reduction in the number of hospitals improve the quality and lower the cost of medical care? Is the cost of health care being inflated by the purchase of medical equipment to fill each hospital? Is the equipment being used inappropriately to justify its purchase?

Research: Is there enough government support for research?

National Societies: Are the national neurosurgical societies strong? Are they respected by the government and people as speaking for neurosurgeons? Do they have power?

\section{Principles of Politics}

In politics there are some simple rules which neurosurgeons must learn if they are to succeed in the political arena where these problems will be solved. The first rule of politics is:

You do something for me, and I will do something for you.

The second rule of politics, and the most difficult for a neurosurgeon to learn is:

Be satisfied with $50 \%$, and come back for the rest later.

You cannot get everything you want immediately. You must compromise but persist to gain what you desire!

\section{Goals for Medicine}

What we need as a goal in medicine throughout the world is to provide the best health care for the largest number of people at the lowest cost. This goal is the most practical, reasonable, yet idealistic goal we can all strive to achieve. It is the essence of the profession of medicine. When we sell our medical souls to the business principle of making money, the public's respect for the profession of medicine will sink to the level of its disrespect for the business community. The profession of medicine and all it means to us and our patients will be lost. We must never lose sight of the principle that

THE PATIENT COMES FIRST!

\section{Possible Solutions for Health Care Problems Facing Japanese Neurosurgeons}

What, then, are possible solutions to the problems 
listed above?

The national neurosurgical societies should form a Planning Committee to evaluate the problems facing neurosurgery. The Planning Committee would a) consult economists to develop economic forecasts for the future of medicine, neurosurgery, and health care for Japan; b) consult business leaders and lawyers to obtain advice on organizing as a society to become politically influential; c) visit other countries to learn how other neurosurgeons and specialists have solved similar problems; d) work with representatives of the government as partners in solving these problems; and e) utilize the talent and connections of the neurosurgeons in Japan who are influential in the business and political community to advance the goals of neurosurgery. The Planning Committee should develop a strategic plan for the improvement of health care and neurosurgery in Japan.

Care of patients with complex problems should be centralized in The Japan Neurosurgical Societyapproved or officially recognized hospitals. This reorganization, although politically difficult, should provide better quality of care and better training of physicians while lowering the cost of health care. Selected hospitals should be closed or merged to reduce the costs of care and improve its quality.

The neurosurgical societies should develop an official training program for the education of neurosurgical residents. For a hospital to qualify for such a training program, it must comply with all of the requirements decided upon by the societies. Residents should undergo regular examinations to verify that they are progressing satisfactorily in their training. There should be a specified operative experience each resident must obtain to reach a level of competence.

More rehabilitation hospitals should be formed so that patients can recover more quickly in specialized facilities dedicated to rehabilitation. As a result, patients will return to work sooner and spare the costs of continued compensation and lost work.

There should be more government support for research. The money saved from the above reorganizations could be spent on research and improving health care.

Public health education needs to be improved. Neurosurgeons should become involved in the education of the public about health problems related to the nervous system. For example, posters on the recognition and prevention of a "brain attack" are seen in a few public areas but not widely even in hospitals. The Internet can be used to provide information to the public about diseases of the nervous system. In the U.S.A., the American Association of
Neurological Surgeons maintains a web site in which the public can obtain information about nervous system diseases; however, The Japan Neurosurgical Society has posted only professional information on its web site. Perhaps this difference may be related to the number of personal computers in the public domain, which is lower in Japan than in the U.S.A. Neurosurgeons can appear on television or be heard on the radio discussing various health matters. Physicians in other disciplines can be educated by neurosurgeons in a planned effort to improve medical professionals knowledge of our specialty.

The neurosurgical societies should become more powerful. It is probably inefficient and economically unjustified to have more than one national neurosurgical society representing neurosurgeons. This single society needs the support of all neurosurgeons and should represent the voice of neurosurgery in Japan. It is crucial that the leadership of this society be those people who are best qualified to hold this position. This is the most important decision to be made; it will affect the outcome of all of this planning. If the appropriate leaders are not selected, neurosurgeons and their patients will ultimately suffer and the goals of the strategic plan will never be achieved.

The national society (or societies) needs to discuss these problems and their solutions at the local and national meetings where all can participate. Neurosurgeons must be educated to work as a group to achieve their goals. Each neurosurgeon should be required to devote one hour a week to the national neurosurgical society to maintain membership in the society and be recognized as a certified neurosurgeon. He/she must contribute to the plans and programs of the society for the improvement of neurosurgery in Japan. This required service would include committee meeting work, participation in public education, or other activities considered essential to the society's strategic plan. Ultimately every neurosurgeon must understand that they must participate in solving the health care problem and also helping neurosurgery and their own careers.

We do not claim to be experts in the solution of these difficult health care problems facing us. These suggestions are offered to stimulate discussion of solutions among Japanese neurosurgeons. These suggestions should lead to better quality of health care, better neurosurgical care, lower costs of health care, better income for qualified neurosurgeons, and reduced malpractice claims.

So, what is your plan for 1999,2000 , and 2001 ? Do you have one? Is it worthwhile to consider that the world is changing and that you must change with it? 
Is it important to have a plan for your future or should you leave it to the politicians and business people to make one for you? The choice is up to you!

\section{Reference}

1) Fukui M: [Keynote announcement of analysis of the present situation of neurosurgery in Japan]. Neurol Med Chir (Tokyo) 38: 305-308, 1998 (Jpn)
Address reprint requests to: J. I. Ausman, M.D., Professor and Head, Department of Neurosurgery (MC 799), College of Medicine, Neuropsychiatric Institute, University of Illinois at Chicago, 912 South Wood Street, Chicago, IL 60612-7329, U.S.A.

(This article was presented as an invited lecture at the 57th Annual Meeting of The Japan Neurosurgical Society, held in October 1998, Sapporo, Japan.) 